\title{
Informatik-Beratung vom FMH Consulting Services Partner
}

Sie sind unzufrieden mit Ihrem IT-System! Soll ein neues System eingeführt werden?

Ihr Archiv hat keinen Platz mehr! Ist es Zeit für die elektronische Krankengeschichte?

Der Aufwand für die Administration ist enorm! Sind die Prozesse in der Praxis noch effizient?

Mit der angemessenen Informatik lassen sich Kosten und Ärger einsparen!

Wir unterstützen und beraten Sie produkteunabhängig und neutral. Umfassendes praktisches und theoretisches Wissen und langjährige Erfahrung aus Arbeiten auf Kunden- und Lieferantenseite ermöglichen uns eine optimale Kundensicht. Projekterfahrung mit verschiedenen Anbietern und Sys-

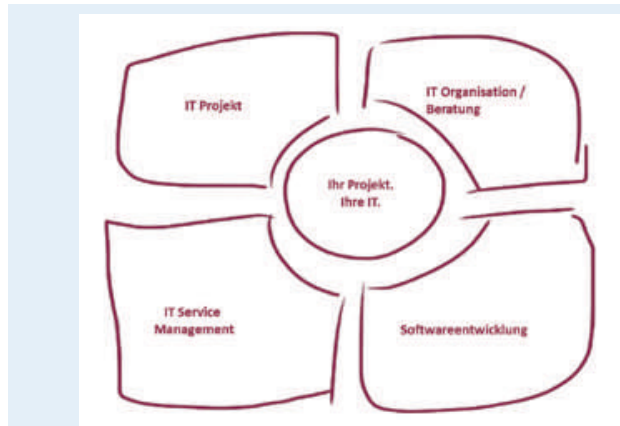

Ihr

Informatik-

Projektpartner

XWare $\mathrm{GmbH}$

Allee $1 \mathrm{~b}$

CH-6210 Sursee

Tel. $\quad+41419221640$

Mobile +41794564366

Web xwr.ch

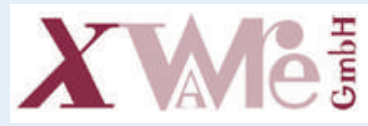

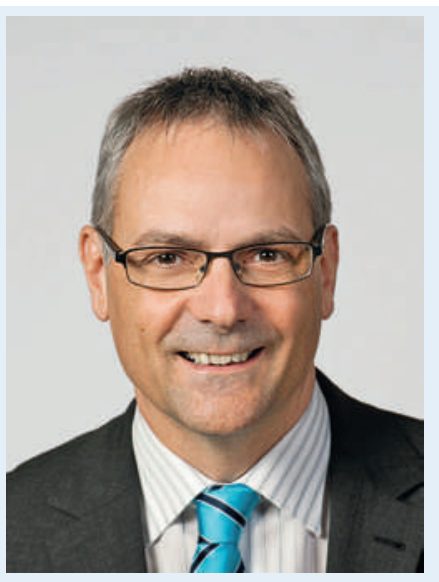

Peter Leuenberger

Eidg. Dipl. Wirtschaftsinformatiker

peter.leuenberger@xwr.ch

Über 20 Jahre Erfahrung in IT-Projekten und IT-Beratung temumgebungen ergänzen das vorhandene Knowhow. Wir weisen Referenzen im Gesundheitswesen, KMU- wie auch im Grosskunden-Umfeld aus, was uns eine differenzierte Betrachtungsweise in den Projekten ermöglicht.

Wir sind bei Ihnen im Einsatz, wenn:

- externe Projekterfahrung und Know-how nötig sind;

- ein Ressourcenengpass eingetreten ist;

- eine ausserordentliche, kritische Projektsituation vorhanden ist;

- eine unabhängige, externe Sicht (Zweitmeinung) gewünscht ist.

\section{Unser Angebot}

- Neutrale, unabhängige Beratung

- Pflichtenheft zugeschnitten auf Ihre Bedürfnisse

- Evaluieren der passenden Lösung

- Koordination aller Beteiligten

- Projektleitung bei Informatikprojekten

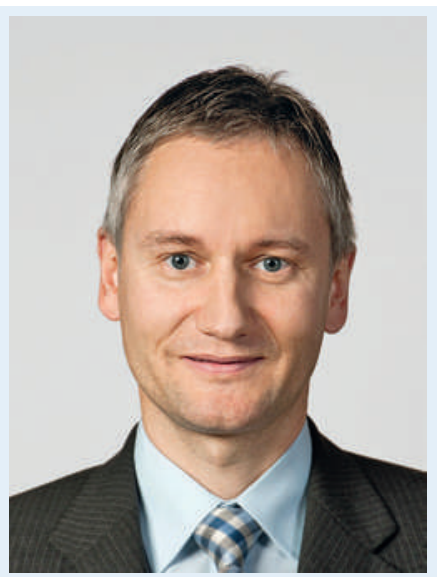

René Brogli

Dipl. Software Ing. FH

rene.brogli@xwr.ch

Über 20 Jahre Erfahrung in IT-Projekten und IT-Beratung 